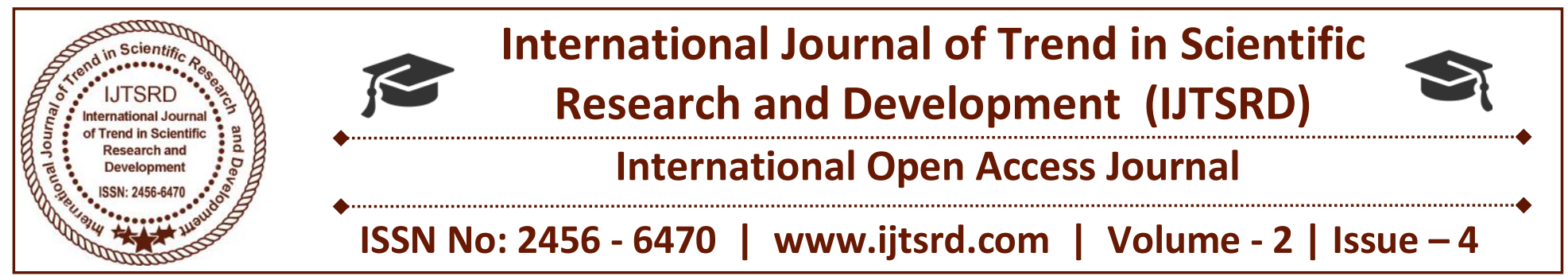

\title{
Real-Time Tracking and Fuel Monitoring System for Vehicle
}

\author{
Yashpalsinh Gohil, Jay Desai \\ Vadodara Institute of Engineering, Vadodara, Gujarat, India
}

Abstract-- In today's world, the actual record of fuel filled and fuel consumption in vehicles is not maintained. It results in a financial loss. To avoid this we are implementing a microcontroller based fuel monitoring and vehicle tracking system. After visited several software-based companies we decide to work on embedded technology. Which is the combination of software as well as hardware? In our project, we are using GPS as well as GSM module. GPS receive the data from satellite and GSM module transmit through SMS with the predefined number both this module work UART (Universal Asynchronous retransmitter protocol). Also, we are using Arduino UNO. It is a single chip microcontroller using this we control all the hardware connected with our project. So we refer the datasheet of 8 mega 328 IC. In 8 mega 328 microcontrollers, there is 2 kilobyte RAM and 32 kilobytes fresh ROM. RAM generally used for defining the data types. While fresh ROM is used to store the program. In this current session, we work UAR protocol programming. In ARDUINO microcontroller try to load simple basic LED on the program using ARDUINO compiler. In this system, we are using both simulations of GSM and GPS module and also we are creating an android application to show all the details of collecting information in an android application.

Keywords-- Arduino UNO, GSM, GPS, Obstacle Sensor, Limit Switch.

\section{INTRODUCTION}

The application of Manage the wheels is mainly attended to monitor multiple vehicles simultaneously. Although most of apply GPS to monitor vehicles, there is no currently available system that can provide real-time fuel consumption information and calculation service. For the aim of real-time monitoring and dynamic mobile data-recording, this study developed a fuel consumption calculating system, which combined Vehicle Tracking System (VTS), GPRS/3G, GPS and Web Server by using Machine to Machine (M2M) framework. In this design, the VTS could extract the necessary information from the vehicles, and with GPRS/3G and GPS techniques, it could achieve both data instantaneity and location preciseness [2].

In addition, according to the Data Collector (DC) and Web of FMS Management Server (MS), this system could provide simple, convenient and fast service for the fleet managers to check the instant data online.

Recent years, the International Crude Oil Price is often fluctuated, which results in the high fuel price. Therefore, a system that could provide fuel consumption calculation and analysis is highly essential for the fleet managers. People increasingly rely on intelligent living style due to the maturation of GPS technique and the rapid development and improvement of communication technology. The technologies are also improving the efficiency of enterprise management, in which the fuel measurement is one of the benefited systems.

Before the GPRS/3G and GPS technique applied in fuel measurement, the fleet managers could only record calculates and analysis car-driving data by paper-works, the calculation, analysis and data proof of fuel consumption were the most important parts and advantages.

Instead of accuracy the two most important things have been to avoid rapid changes in the fuel level displayed and the meter must indicate that the tank is empty when the fuel level is below a predefined level. 


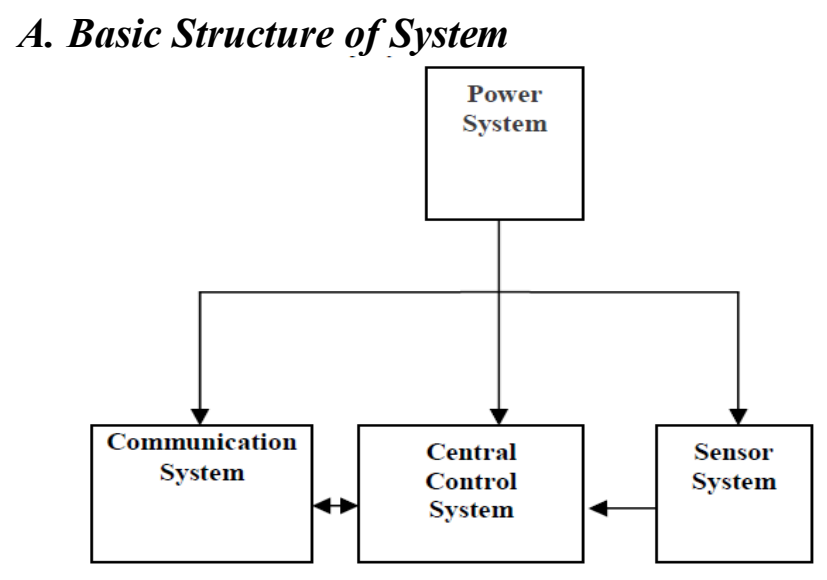

Figure 1: Basic Structure of the system

Basically, the system is composed of the central control System, communication system, sensor system and power system. The system structure is shown in the figure.

\section{Communication System}

Communication system can communicate with Arduino UNO. The first channel uses 8 mega 328 IC; the second one is the obstacle sensor communication system which can measure RPM. The last one uses wireless GPS for tracking location along with the vehicle properly and communication distance can be extended greatly.

\section{Sensor System}

Sensor System is composed of measuring RPM with show fuel level using obstacle sensor.

\section{Power System}

The central control system is powered by DC power supply with proper specifications and the communication system. I.e. GPS and sensor system is also powered by this power supply.

\section{Central Control System}

This is the heart of the monitoring system. It consists of a microcontroller with appropriate interfacing with other devices. It performs all the control actions required for proper operation of all the system.

\section{A. Structure of a unit}

The unit is placed inside the vehicle to sense the fuel level at various time instances and it also tracks the vehicle with help of GPS. To achieve these things the system is equipped with obstacle sensor along with Arduino UNO, GSM, and 8 mega 328 IC as main building blocks of our system.

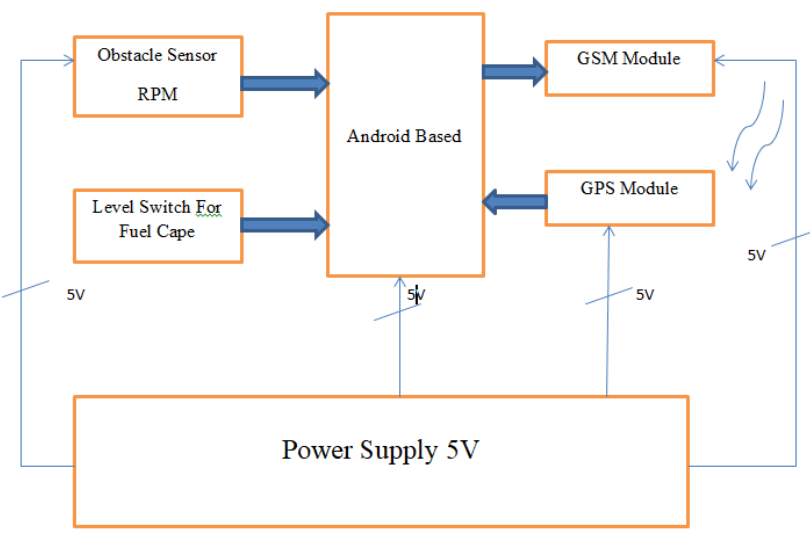

Figure 2: Block Diagram

The 8 mega 328 IC is the heart of our system.

Arduino UNO is the electronic device which contains processing Power, memory and IO ports to interact with different connected devices. In this system 8 mega 328 IC is the brain of the system which stores the status of fuel level in a fuel tank and position of a vehicle. The system is powered by

DC power supply with proper specifications. This supply can be provided by batteries. Obstacle Sensor will be used to sense the measure of RPM along with a quantity of fuel consumed and notify microcontroller about the level of fuel in the fuel tank. By counting this RPM and dividing it by a flow factor we will get the exact amount of fuel filled.

\section{SYSTEM IMPLEMENTATION Interfacing Circuit}

The interfacing circuit consists of 8 mega 328 IC, GSM, GPS, obstacle sensor, DC motor, Limit switch, $16 \times 2$ LCD.

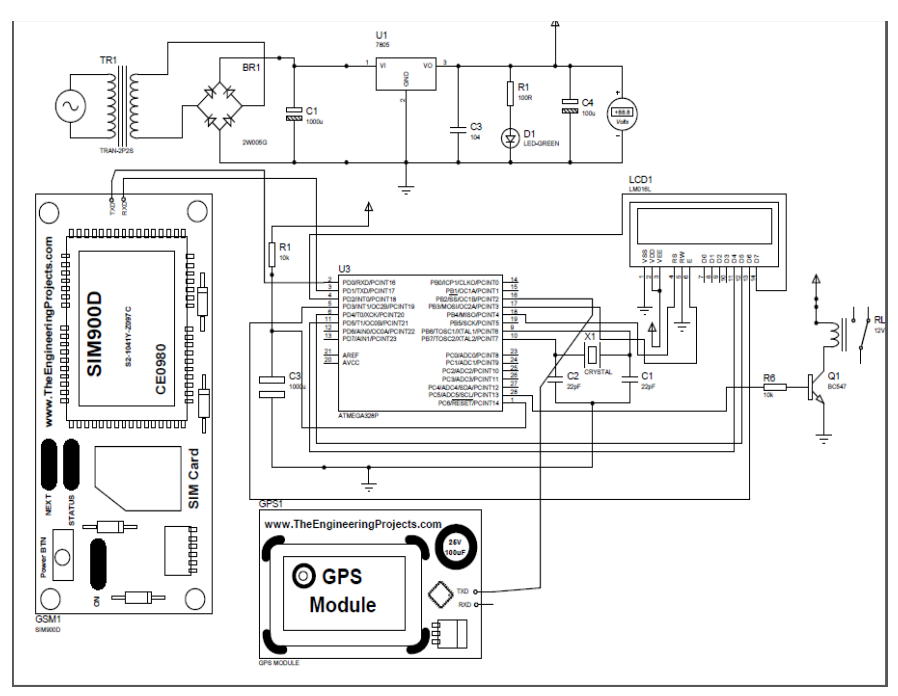

Figure 3: Interfacing Diagram

The GPS module continuously produces a set of data 
regarding the position of the earth surface where it is situated which includes the current position with respect to the equator of the earth in terms of Latitude and Longitude. This data can be decoded and printed into the readable format with the help of a microcontroller only. In this project, the data regarding the geographical coordinate is extracted from the GPS output with the help of the Arduino. The Arduino can be used as a stand-alone board of which the output or inputs can be taken from the boards or given to the board. They can communicate using standard communication ports like USART, TWI, and SPI etc. which enables them to be connected with various kinds of devices. The Arduino board is designed for easy prototyping and the IDE used for coding is very simple and provides so many libraries for interfacing with common external devices.

A GSM Module is basically a GSM Modem (like SIM 300) connected to a PCB with different types of output taken from the board - say TTL Output (for Arduino, other microcontrollers) and RS232 Output to interface directly with a PC (personal computer) [5]. The board will also have pins or provisions to attach mic and speaker, to take out $+5 \mathrm{~V}$ or other values of power and ground connections.

LCD modules form a very important part of many Arduino based embedded system designs. So the knowledge on interfacing LCD module to Arduino is very essential in designing embedded systems. This section of the article is about interfacing an Arduino to $16 \times 2 \mathrm{LCD}$. JHD162A is the LCD module used here [4]. JHD162A is a $16 \times 2$ LCD module based on the HD44780 driver. The JHD162A has 16 pins and can be operated in 4-bit mode (using only 4 data lines) or 8-bit mode (using all 8 data lines). Here we are using the LCD module in 4-bit mode. First, I will show you how to display plain text messages on the LCD module using Arduino.

\section{CONCLUSION}

This project will grow features and facility in the transportation field. Many new features are being added to enhance the monitoring and tracking operations using recent technologies. Our attempt is to design the best prototype for the same. The system helps the owner of the vehicle who is at a remote location to perform the tasks of detecting the fuel theft and tracking the vehicle accurately and measuring RPM continuously. Many factors of the transportation system are considered. It can work in various environments.

\section{References}

[1] NMEA Reference Manual SiRF Technology, Inc.148 East Brokaw RoadSan Jose, CA 95112 U.S.A. Available: http://www.nmea.org.

[2] Yin-Jun Chen, Ching-Chung Chen, Shou-Nian Wang, Han-En Lin, Roy C. Hsu GPSenseCar -A Collision Avoidance Support System Using RealTime GPS Data in a Mobile Vehicular Network" 0-7695-2699- 3/06/\$20.00 (c) IEEE.

[3] Jerry Luecke, Analog and Digital Circuits for Electronic Control System Applications, Using the TI MSP430 Microcontroller", Elsevier: Newnes publications, Burlington, MA 01803, USA, 2005.

[4] Pankaj Verma, J.S Bhatia "Design and Development of GPS-GSM based Tracking System with Google map based Monitoring", International Journal of Computer Science, Engineering and Applications (IJCSEA) Vol.3, No.3, June 2013.

[5] Fleischer, P.B.; Nelson, A.Y.; Sowah, R.A.; Bremang, A., "Design and development of GPS/GSM based vehicle tracking and alert system for commercial inter-city buses," Adaptive Science \& Technology (ICAST), 2012 IEEE 4th International Conference on, vol., no., pp.1, 6, 2527 Oct. 2012.

[6] El-Medany,W.; Al-Omary,A.; Al-Hakim,R.; AlIrhayim,S.; Nusaif, M., "A Cost-Effective RealTime Tracking System Prototype Using Integrated GPS/GPRS Module," Wireless and Mobile Communications (ICWMC), 2010 6th International Conference on,vol.,no.,pp.521,525,20-25 Sept.2010.

[7] William J. Fleming- Life senior member IEEE "New Automotive Sensor- a Review" IEEE Sensor Journal, vol. 8, no. 11, November 2008.

[8] Moe Rahnema "Overview of GSM System and Protocol Architecture" IEEE Communication Magazine, vol. 10, no. 11-14, April 2003.

[9] William J. Fleming- Life senior member IEEE "Overview of Automotive Sensors" IEEE Sensor Journal, vol. 1, no. 4, December 2001.

[10] Hu Jian-ming; Li Jie; Li Guang-Hui, "Automobile Anti-theft System Based on GSM and GPS Module," Intelligent Networks and Intelligent Systems (ICINIS), 2012 Fifth International Conference on, vol., no., pp.199, 201, 1-3 Nov. 2012 